Apuntes Universitarios, 2021: 11(2), abril-junio ISSN:

2304-0335 DOI: https://doi.org/10.17162/au.v11i2.631

\title{
Burnout académico en estudiantes universitarios peruanos
}

\author{
Academic burnout in Peruvian university students \\ Edwin Gustavo Estrada Araoz ${ }^{11}$; Néstor Antonio Gallegos Ramos ${ }^{2}$ \\ Helen Juddy Mamani Uchasara ${ }^{3}$ \\ Universidad Nacional Amazónica de Madre de Dios ${ }^{123}$ \\ ORCID ID: https://orcid.org/0000-0003-4159-934X ${ }^{1}$ \\ ORCID ID: https://orcid.org/0000-0003-1436-9207² \\ ORCID ID: https://orcid.org/0000-0003-1735-8570³
}

Recibido: 15 de setiembre de 2020

Aceptado: 29 de noviembre de 2020

\section{Resumen}

La presente investigación tuvo como objetivo describir el burnout académico de los estudiantes de la carrera profesional de Educación de una universidad pública de Madre de Dios (Perú). El enfoque de investigación fue cuantitativo, el diseño no experimental, y de tipo descriptivotranseccional. La muestra fue conformada por 202 estudiantes a quienes se les aplicó un cuestionario de datos sociodemográficos y académicos así como el Cuestionario Maslach Burnout Inventory Student Survey (MBI-SS), instrumento con adecuados niveles de validez y confiabilidad. Los hallazgos indican que los estudiantes se caracterizan por presentar niveles moderados de burnout académico y se determinó que algunas variables como el sexo, el grupo etario, la situación laboral y el año de estudio se asocian significativamente con el burnout académico. Finalmente se concluyó que es necesario que se diseñen y ejecuten programas que desarrollen los factores protectores de los estudiantes para que puedan afrontar adecuadamente situaciones adversas y estresantes y se disminuya así la prevalencia del burnout académico.

Palabras clave: Burnout académico; agotamiento emocional; eficacia académica; cinismo; educación superior universitaria.

\footnotetext{
Abstract

The present research aimed to describe the academic burnout of the students of the professional education career of a public university in Madre de Dios (Peru). The research approach was

${ }^{1}$ Correspondencia al autor:

E-mail: edwin5721@ outlook.com
} 
quantitative, the design was non-experimental, and the type was descriptive-transectional. The sample consisted of 220 students to whom a sociodemographic and academic data questionnaire was applied as well as the Maslach Burnout Inventory Student Survey (MBI-SS), an instrument with adequate levels of validity and reliability. The findings indicate that students are characterized by moderate levels of academic burnout and it was determined that some variables such as sex, age group, work situation and year of study are significantly associated with academic burnout. Finally, it was concluded that it is necessary to design and implement programs that develop the protective factors of students so that they can adequately cope with adverse and stressful situations and thus decrease the prevalence of academic burnout.

Keywords: Academic burnout; emotional exhaustion; academic effectiveness; cynicism; university higher education.

\section{Introducción}

El síndrome de burnout, también conocido como el síndrome del trabajador quemado, fue inicialmente descrito por Freudenberger (1974) en la década de los setenta y desarrollado a profundidad por Maslach (1982) una década después. Se refiere a una forma de cansancio o agotamiento provocado principalmente por la labor que realizan los profesionales que tienen entre sus responsabilidades interactuar con personas, como es el caso de los docentes, médicos, policías, enfermeras, psicólogos, entre otros (Arias et al., 2017; Aguayo et al., 2019; Carlotto, Gonçalves y Brazil, 2005). Por ello, en las últimas décadas ha crecido el interés por investigar dicha variable debido a las repercusiones que presenta en la salud física y psicológica de las personas así como su entorno familiar, laboral y social (Martínez, 2010; De la Rosa et al., 2015).

Sin embargo, el concepto del burnout se amplió a otros ámbitos, pues se determinó que se puede presentar en cualquier circunstancia, es decir, que trasciende a otros contextos, siendo uno de ellos, el académico (Rodríguez et al., 2019; Correa et al., 2019; García et al., 2018). Ello debido a que en el contexto estudiantil de la educación universitaria existen exigencias académicas (exámenes, tareas, informes, exposiciones, etc.) que puedan afectar de manera negativa el ajuste psicosocial de los estudiantes y, por ende, sobre su salud mental. Asimismo, la situación puede agravarse en el caso de las exigencias sean excesivas y que el estudiante no haya desarrollado estrategias para enfrentarlas (Merino y Fernández, 2020).

Por otra parte, esta condición también puede agudizarse debido a que no siempre las universidades fomentan el desempeño académico del estudiante, como el no proveer suficientes recursos, las pésimas estrategias didácticas de los docentes, la existencia de dinámicas negativas 
para la realización del trabajo académico y excesivas exigencias (Loayza et al., 2016; Osorio, Parrello y Prado, 2020).

Así pues, el burnout académico fue definido como una consecuencia y respuesta al estrés crónico y se vincularía a la labor, la actividad y el entorno académico, de carácter negativo, dañino y que podría influir en el desenvolvimiento, la identificación y aceptación de los estudiantes con su formación y vida académica así como en su salud mental (Caballero, Breso y Gonzáles, 2015). Lo expuesto implica un sentimiento de agotamiento emocional experimentado por los estudiantes originado por las demandas académicas, provocando a su vez una actitud cínica, indiferente y poco comprometida con sus responsabilidades académicas, además de creencias de poca capacidad y autoeficacia en las actividades que desarrolla (Domínguez, 2018; Aguayo et al., 2019; Marenco, Suárez y Palacio, 2017).

Pues bien, es necesario remarcar que el burnout académico puede ocasionar graves consecuencias, tanto físicas como psicológicas para la salud de los estudiantes. "Estas consecuencias podrían ser psicosomáticas (problemas cardiovasculares, trastornos gastrointestinales, falta de sueño, fatiga), emocionales (insatisfacción con los estudios, depresión, falta de autoestima, desmotivación) o conductuales (bajo rendimiento académico, alcohol o abuso de drogas, mala alimentación, absentismo, abandono escolar)” (Aguayo et al., 2019, p.708).

Existen variables personales, académicas y sociodemográficas que se asocian al burnout académico. A partir de estudios empíricos o abordajes teóricos pueden clasificarse, según su naturaleza, en variables del contexto académico (comunicación y distanciamiento con el docente, estrategias y exigencia del docente, incompatibilidad con la carga horaria, etc.), variables del contexto social (competitividad entre compañeros, participación en actividades extracurriculares, carrera profesional elegida, nivel socioeconómico, etc.) y variables intrapersonales (sexo, gestión del tiempo, ansiedad ante los exámenes, autoeficacia, rasgos de la personalidad, etc.) (Caballero, Hederich y Palacio, 2010).

Ahora bien, se han encontrado estrategias de afrontamiento que los estudiantes tienden a usar para lidiar con el burnout académico. En ese sentido y de acuerdo a Caballero et al. (2015), se pueden clasificar en tres tipos: el primerio, centrado en el problema, la cual busca modificar las exigencias o eventos que provocan altos niveles de estrés, tratando de resolver el problema o reducir su impacto. El segundo tipo está orientado al afrontamiento centrado en las emociones, que conlleva a esforzarse para disminuir o eliminar los pensamientos y sentimientos negativos 
provocados por la situación estresante. Por último, el tercero, tiene que ver con el afrontamiento de evitación, que implica eludir la situación estresante dejando de pensar en ella o distrayéndose con otras actividades o acciones.

Existen diversos estudios que investigaron acerca del burnout académico en estudiantes universitarios y reportaron que existía una prevalencia considerable, ya sea en moderados o altos niveles (Osorio et al., 2020; Bolaños y Rodríguez, 2016; Loayza et al., 2016; Marenco et al., 2017; Núñez, 2017; Estrada et al., 2017; Uribe y Illesca, 2017; Rodríguez et al., 2019). Del mismo modo, algunas investigaciones buscaron conocer qué variables sociodemográficas, académicas o laborales se asocian al burnout académico y, aunque algunos hallazgos no son totalmente concluyentes, determinaron que el ser mujer (Osorio et al., 2020; Bolaños y Rodríguez, 2016; Caballero et al., 2010; Uribe y Illesca, 2017; Rodríguez et al., 2019), pertenecer a los grupos etarios más jóvenes (Bolaños y Rodríguez, 2016; Aguayo et al., 2019; Estrada et al., 2017), cursar el primer y último año de estudios (Bolaños y Rodríguez, 2016; Aguayo et al., 2019; Estrada et al., 2017; Uribe y Illesca, 2017) y trabajar y estudiar a la vez (Caballero, 2006) favorecería a la aparición del burnout académico en el transcurso de la vida universitaria.

La presente investigación es relevante pues permitirá conocer cuál es la prevalencia del burnout académico en una universidad pública de Madre de Dios (Perú). A nivel local y regional son muy pocas las investigaciones que han estudiado dicha enfermedad por lo que resulta importante estudiar cómo afecta el burnout académico y a partir de los hallazgos que se encuentren se podrán diseñar y ejecutar acciones preventivas o correctivas con la finalidad de lograr disminuir su prevalencia y generar las condiciones para que los estudiantes se puedan desenvolver adecuadamente en la universidad, mejorando su calidad de vida y bienestar psicológico para que puedan afrontar sus estudios de mejor manera. Por lo expuesto, el objetivo de la presente investigación fue describir el burnout académico de los estudiantes de la carrera profesional de Educación de una universidad pública de Madre de Dios (Perú).

\section{Metodología}

El enfoque de investigación fue cuantitativo, pues se realizó la recolección y el análisis de datos haciendo uso de la estadística para determinar el comportamiento de la muestra (Sánchez, Reyes y Mejía, 2018). En cuanto al diseño, fue no experimental, pues la variable de estudio no fue manipulada intencionalmente, sino fue observada $y$ finalmente, el tipo fue descriptivo 
transeccional, ya que ya que se analizaron las propiedades y características de la variable burnout académico y la recolección de datos se dio en un solo momento (Carrasco, 2006).

Con relación a la población, fue conformada por 423 estudiantes pertenecientes a la carrera profesional de Educación, en sus tres especialidades: Inicial y Especial, Primaria e Informática y Matemática y Computación, matriculados en el ciclo 2020-II y la muestra quedó constituida por 202 estudiantes, cantidad que fue estimada mediante un muestreo no probabilístico, por conveniencia.

La técnica utilizada fue la encuesta y los instrumentos para recoger los datos fueron el cuestionario de datos sociodemográficos y académicos y el Cuestionario Maslach Burnout Inventory Student Survey (MBI-SS). Ambos cuestionarios fueron estructurados a través de la herramienta digital Google Forms. Por otro lado, el cuestionario de datos sociodemográficos y académicos fue elaborado por los autores con la finalidad de recoger información referida específicamente al sexo, el grupo etario, la especialidad, el promedio ponderado, el año de estudios, la situación laboral y el estado civil.

Con relación al Cuestionario Maslach Burnout Inventory Student Survey (MBI-SS), fue diseñado originalmente por Maslach y Jackson (1981), posteriormente adecuado al ámbito académico por Schaufeli et al. (2002) y finalmente adaptado a la realidad peruana por Correa et al. (2019). Evalúa el agotamiento originado por las demandas del estudio, las actitudes de desinterés frente a las actividades académicas, las dudas acerca del valor del estudio así como su sentimiento de incompetencia en su rol de estudiantes. Está conformado por 15 ítems de tipo Likert (nunca, pocas veces, a veces, casi siempre y siempre) distribuidos en 3 dimensiones: el agotamiento emocional, el cinismo y la eficacia académica. Sus propiedades psicométricas fueron determinadas a través del proceso de validación y confiabilidad. La validación de contenido se realizó mediante la técnica de juicio de expertos, obteniéndose luego de concordancia un coeficiente V de Aiken de 0,838; lo que indica que el cuestionario tuvo buena validez (Ruiz, 2002). Respecto a la confiabilidad, fue obtenida a través una prueba piloto realizada a 35 estudiantes, obteniéndose un coeficiente Alfa de Cronbach de 0,894 para la variable y coeficientes de 0,821;0,843 y 0,899 para las dimensiones agotamiento emocional, cinismo y eficacia académica respectivamente, lo que significa, según Ruiz (2002), que el instrumento, a nivel general, y sus factores poseen muy alta confiabilidad. 
El proceso de recolección de datos se efectuó en el mes de agosto del año 2020 y para ello se puso en contacto con los estudiantes mediante el uso de la red social digital Whatsapp con la finalidad de indicarles el propósito de la investigación y enviarles el enlace para que puedan acceder a Google Forms. Cuando ellos accedieron al mencionado formulario, leyeron con atención las orientaciones y brindaron su consentimiento informado, admitiendo que participaron de la investigación de manera voluntaria. Posteriormente, desarrollaron el cuestionario, el cual tuvo una duración aproximada 15 minutos. El acceso a la encuesta fue cerrado una vez que se obtuvieron 202 respuestas, la información obtenida fue exportada a un archivo de Microsoft Excel y se realizó, en el caso del Cuestionario Maslach Burnout Inventory Student Survey (MBI-SS), la calificación teniendo en cuenta su escala de valoración.

Para efectuar el análisis estadístico se recurrió al Software SPSS versión 22, donde los resultados descriptivos fueron sistematizados mediante tablas de frecuencia y porcentaje. Por otro lado, la parte inferencial se realizó utilizando la prueba no paramétrica Chi Cuadrado con la finalidad de conocer si el burnout académico se asociaba significativamente con las variables propuestas.

\section{Análisis de datos}

En la tabla 1 se describen las características sociodemográficas y académicas de la muestra. Se puede ver así que la edad promedio de los participantes fue 22 años, la mayoría de participantes fueron mujeres (59,9\%), sus edades oscilaban entre 16 y 20 años (44\%), pertenecían a la especialidad de Inicial y Especial (46,1\%), tenían un promedio ponderado entre 11 y 14 (50\%), cursaban el segundo año de estudios $(29,2 \%)$, no trabajaban $(52 \%)$ y tenían una pareja estable $(54,5 \%)$. 
Tabla 1

Características de la muestra

\begin{tabular}{llcc}
\hline & Variables & $\mathrm{n}=202$ & $\%$ \\
\hline Edad media & Masculino & $22,8 \pm 5,989$ & \\
Sexo & Femenino & 81 & 40,1 \\
& De 16 a 20 años & 121 & 59,9 \\
\multirow{4}{*}{ Grupo etario } & De 21 a 25 años & 89 & 44,0 \\
& De 26 a 30 años & 67 & 33,2 \\
& De 31 a más años & 29 & 14,4 \\
Especialidad & Inicial y Especial & 17 & 8,4 \\
& Primaria e Informática & 93 & 46,1 \\
& Matemática y Computación & 74 & 36,6 \\
Promedio ponderado & 35 & 17,3 \\
& Menos de 11 & 5 & 2,5 \\
& De 11 a 14 & 101 & 50,0 \\
& De 15 a 17 & 65 & 32,2 \\
Año de estudio & De 18 a 20 & 31 & 15,3 \\
& Primero & 33 & 16,3 \\
Situación laboral & Segundo & 59 & 29,2 \\
Estado civil & Tercero & 48 & 23,8 \\
& Cuarto & 38 & 18,8 \\
& Quinto & 24 & 11,9 \\
& Trabaja & 97 & 48,0 \\
& No trabaja & 105 & 52,0 \\
& Con pareja & 110 & 54,5 \\
& Sin pareja & 92 & 45,5 \\
\hline
\end{tabular}

Fuente: Base de datos.

Los resultados expuestos en la tabla 2 revelan que la mayoría de estudiantes presentan niveles moderados de burnout académico $(52,5 \%)$. En cuanto a sus dimensiones, los estudiantes también se caracterizan por tener niveles moderados de agotamiento emocional $(66,3 \%)$, cinismo $(43,1 \%)$ y eficacia académica $(36,1 \%)$. 
Tabla 2

Resultados descriptivos del burnout académico y sus dimensiones

\begin{tabular}{lcccccccc}
\hline \multirow{2}{*}{ Niveles } & \multicolumn{2}{c}{ Burnout } & \multicolumn{2}{c}{ Agotamiento } & \multicolumn{2}{c}{ Cinismo } & \multicolumn{2}{c}{ Eficacia } \\
& \multicolumn{2}{c}{ académico } & \multicolumn{2}{c}{ emocional } & \multicolumn{2}{c}{ académica } \\
& $\mathrm{n}$ & $\%$ & $\mathrm{n}$ & $\%$ & $\mathrm{n}$ & $\%$ & $\mathrm{n}$ & $\%$ \\
\hline Alto & 51 & 25,2 & 33 & 16,4 & 54 & 26,7 & 61 & 30,2 \\
Moderado & 106 & 52,5 & 134 & 66,3 & 87 & 43,1 & 73 & 36,1 \\
Bajo & 45 & 22,3 & 35 & 17,3 & 61 & 30,2 & 68 & 33,7 \\
Total & 202 & 100,0 & 202 & 100,0 & 202 & 100,0 & 202 & 100,0 \\
\hline
\end{tabular}

Fuente: Base de datos

En la tabla 3 se observa que el sexo, el grupo etario, el año de estudio y la situación laboral de los estudiantes se asocian significativamente con la prevalencia del burnout académico $(p<0,05)$, sin embargo, no se encontraron asociaciones significativas con la especialidad, el promedio ponderado y el estado civil ( $>>0,05)$. Así pues, se pudo determinar que estudiantes del sexo femenino, que tenían entre 16 a 20 años, que estaban cursando los primeros años de estudio y trabajaban presentaron niveles de burnout académico ligeramente superiores a los demás grupos. 
Tabla 3

Burnout académico y variables sociodemográficas y académicas

\begin{tabular}{|c|c|c|c|c|c|c|}
\hline \multirow{2}{*}{\multicolumn{2}{|c|}{ Variables }} & \multicolumn{3}{|c|}{ Burnout académico } & \multirow{2}{*}{$X^{2}$} & \multirow{2}{*}{ Sig. } \\
\hline & & Alto & Moderado & Bajo & & \\
\hline \multirow{2}{*}{ Sexo } & Masculino & $15(18,5 \%)$ & $44(54,3 \%)$ & $22(27,2 \%)$ & \multirow{2}{*}{21,335} & \multirow{2}{*}{0,000} \\
\hline & Femenino & $36(29,8 \%)$ & $57(47,1 \%)$ & $28(23,1 \%)$ & & \\
\hline \multirow{4}{*}{ Grupo etario } & De 16 a 20 años & $25(28,1 \%)$ & $47(52,8 \%)$ & $17(19,1 \%)$ & \multirow{4}{*}{28,568} & \multirow{4}{*}{0,000} \\
\hline & De 21 a 25 años & $18(26,9 \%)$ & $35(52,2 \%)$ & $14(20,9 \%)$ & & \\
\hline & De 26 a 30 años & $6(20,7 \%)$ & $14(48,3 \%)$ & $9(31,0 \%)$ & & \\
\hline & De 31 a más años & $2(11,8 \%)$ & $10(58,8 \%)$ & $5(29,4 \%)$ & & \\
\hline \multirow{3}{*}{ Especialidad } & Inicial y Especial & $24(25,8 \%)$ & $52(55,9 \%)$ & $17(18,3 \%)$ & \multirow{3}{*}{4,588} & \multirow{3}{*}{0,061} \\
\hline & $\begin{array}{l}\text { Primaria e } \\
\text { Informática }\end{array}$ & $20(27,0 \%)$ & $34(46,0 \%)$ & $20(27,0)$ & & \\
\hline & $\begin{array}{l}\text { Matemática y } \\
\text { Computación }\end{array}$ & $7(20,0 \%)$ & $20(57,1 \%)$ & $8(22,9 \%)$ & & \\
\hline \multirow{4}{*}{$\begin{array}{l}\text { Promedio } \\
\text { ponderado }\end{array}$} & Menos de 11 & $1(20,0 \%)$ & $3(60,0 \%)$ & $1(20,0 \%)$ & \multirow{4}{*}{8,304} & \multirow{4}{*}{0,054} \\
\hline & De 11 a 14 & $21(20,8 \%)$ & $61(60,4 \%)$ & $19(18,8 \%)$ & & \\
\hline & De 15 a 17 & $17(26,2 \%)$ & $32(49,2 \%)$ & $16(24,6 \%)$ & & \\
\hline & De 18 a 20 & $12(38,7 \%)$ & $10(32,3 \%)$ & $9(29,0 \%)$ & & \\
\hline \multirow{5}{*}{$\begin{array}{l}\text { Año de } \\
\text { estudio }\end{array}$} & Primero & $12(36,4 \%)$ & $11(33,3 \%)$ & $10(30,3 \%)$ & \multirow{5}{*}{7,520} & \multirow{5}{*}{0,008} \\
\hline & Segundo & $14(23,7 \%)$ & $39(66,1 \%)$ & $6(10,2 \%)$ & & \\
\hline & Tercero & $7(14,6 \%)$ & $29(60,4 \%)$ & $12(25,0 \%)$ & & \\
\hline & Cuarto & $9(23,7 \%)$ & $18(47,4 \%)$ & $11(28,9 \%)$ & & \\
\hline & Quinto & $9(37,5 \%)$ & $9(37,5 \%)$ & $6(25,0 \%)$ & & \\
\hline \multirow{2}{*}{$\begin{array}{l}\text { Situación } \\
\text { laboral }\end{array}$} & Trabaja & $31(32,0 \%)$ & $55(56,7 \%)$ & $11(11,3 \%)$ & \multirow{2}{*}{9,983} & \multirow{2}{*}{0,010} \\
\hline & No trabaja & $20(19,0 \%)$ & $51(48,6 \%)$ & $34(32,4 \%)$ & & \\
\hline \multirow{2}{*}{ Estado civil } & Con pareja & $30(27,3 \%)$ & $55(50,0 \%)$ & $25(22,7 \%)$ & \multirow{2}{*}{6,215} & \multirow{2}{*}{0,079} \\
\hline & Sin pareja & $21(22,8 \%)$ & $51(55,5 \%)$ & $20(21,7 \%)$ & & \\
\hline
\end{tabular}

Fuente: Base de datos

\section{Discusión}

Estudiar una carrera universitaria puede ser muy exigente y provocar altos niveles de estrés debido a las altas demandas académicas, sociales y personales. Por ello, existen muchas probabilidades de que los estudiantes padezcan de burnout académico. En ese sentido, en la presente investigación se describió la prevalencia del burnout académico en los estudiantes de la carrera profesional de Educación de una universidad pública de Madre de Dios e identificó algunas variables que se asocian a ella. 
Un primer hallazgo encontrado da cuenta que los estudiantes se caracterizaban por tener niveles moderados de burnout académico, es decir, en algunas oportunidades llegaron a agotarse emocionalmente, al punto de pensar que no pueden dar más de sí mismos, del mismo modo, han perdido el interés en sus estudios, dándole poca importancia y destinándole poco tiempo y finalmente, han llegado a dudar de su eficacia académica, desestimando sus competencias, capacidades y habilidades. Lo expuesto llama la atención ya que la sintomatología del burnout académico no solo afecta directamente su desempeño en la universidad, sino menoscaba su calidad de vida. Los resultados descritos coinciden con diversas investigaciones que reportaron la presencia de burnout académico en los estudiantes, cuyos niveles fluctuaban entre los niveles moderado y alto y sería provocado principalmente por las demandas del contexto universitario, social y familiar (Osorio et al., 2020; Bolaños y Rodríguez, 2016; Loayza et al., 2016; Marenco et al., 2017; Núñez, 2017; Estrada et al., 2017; Uribe y Illesca, 2017; Rodríguez et al., 2019).

Al respecto, Marenco et al. (2017) precisan de no existir factores protectores, estrategias regulativas y de afrontamiento a esta enfermedad, ocasionaría problemas cardiovasculares, desórdenes mentales, hipertensión, trastornos mentales e insomnio, aumentando los niveles de morbilidad.

Respecto a las variables sociodemográficas, se pudo identificar que el síndrome de burnout se asocia significativamente al sexo de los estudiantes $(\mathrm{p}=0,000<0,05)$. En ese sentido, se encontró que las mujeres se caracterizaban por presentar niveles moderados y altos de burnout académico en comparación a los varones, en quienes predominaban los niveles moderados y bajos. Este hallazgo es similar a los resultados reportados por algunas investigaciones que encontraron que las mujeres presentaban niveles ligeramente superiores de burnout académico lo cual sería explicado porque ellas también tienden a sufrir con mayor frecuencia trastornos interiorizantes (depresión y ansiedad) frente a situaciones de estrés provocados en muchos casos por los esfuerzos que realizan para destacar y buscar ser las mejores (Osorio et al., 2020; Bolaños y Rodríguez, 2016; Caballero et al., 2010; Uribe y Illesca, 2017; Rodríguez et al., 2019).

Otro hallazgo bastante revelador es que se pudo determinar que el burnout académico también se asoció de manera significativa con el grupo etario al que pertenecían los estudiantes $(\mathrm{p}=0,000<0,05)$. Así pues, los estudiantes más jóvenes (menores de 26 años) se caracterizaban por tener valores ligeramente superiores de burnout académico, pues presentaban niveles moderados y altos, mientras que en los estudiantes de edades superiores (de 26 a más años) predominaban los 
niveles moderados y bajos. Lo expuesto es corroborado por investigaciones que determinaron que los estudiantes que pertenecían a los grupos etarios de menor edad eran los más afectados por el síndrome de burnout, específicamente por el agotamiento emocional (Bolaños y Rodríguez, 2016; Aguayo et al., 2019; Estrada et al., 2017).

Del mismo modo, se determinó que el burnout académico se asoció de manera significativa con el año de estudios $(\mathrm{p}=0,008<0,05)$. Lo expuesto quiere decir que los estudiantes que cursaban el primero, segundo y quinto año de estudios presentaban valores ligeramente superiores de burnout académico, caracterizándose específicamente por tener niveles alto y moderado, mientras que en los estudiantes que cursaban el tercer y cuarto año predominaban los niveles moderado y bajo. Este hallazgo coincide con lo reportado por algunas investigaciones que determinaron que los alumnos ingresantes y que están a punto de egresar tienden a presentar mayores niveles de burnout académico, debido posiblemente a que, en el caso de los ingresantes, estén adaptándose a la transición de la vida escolar a la vida universitaria y las altas demandas académicas que ella genera. Por otro lado, en el caso de los estudiantes que están en el último año de estudios, tienden a presentar sintomatologías asociadas al estrés y ansiedad debido a que se encuentran elaborando sus trabajos de investigación conducentes a la obtención de su título profesional (Bolaños y Rodríguez, 2016; Aguayo et al., 2019; Estrada et al., 2017; Uribe y Illesca, 2017).

Así también, se encontró que los niveles del burnout académico también están asociados de manera significativa a la situación laboral de los estudiantes $(\mathrm{p}=0,008<0,05)$. Bajo esa premisa, los estudiantes que trabajaban y estudiaban se caracterizaban por presentar niveles ligeramente superiores de burnout académico en comparación a los estudiantes que solo estudiaban. Este hallazgo coincide parcialmente con lo reportado por Caballero (2006) quien encontró que los estudiantes que trabajaban presentaban mayores niveles de eficacia académica, factor que compone el burnout académico. El hallazgo tiene lógica ya que el estudiante al tener que trabajar y estudiar suele preocuparse más por gestionar su tiempo, cumplir sus responsabilidades oportunamente y ello a su vez le genera niveles elevados de estrés.

El burnout académico es una enfermedad bastante común en los estudiantes universitarios y por ese motivo, debe ser detectada o prevenirse pues de presentarse en niveles altos o profundos y no realizar un tratamiento, provocaría síntomas psicosomáticos, conductuales y emocionales (Rosales y Rosales, 2013), que indudablemente afectarán negativamente su rendimiento académico y podrían provocar la deserción de la universidad (García et al., 2018). 
Por último, es necesario señalar que la presente investigación tuvo algunas limitaciones, como la cantidad de la muestra, lo cual no permite generalizar los resultados y los restringe al presente grupo de estudiantes universitarios. Del mismo modo, al ser una investigación transversal, la recolección de datos se realizó al finalizar el ciclo académico, lo cual podría haber afectado los resultados. Finalmente, solo se asociaron las variables sociodemográficas y académicas con el burnout académico pero no con sus factores.

\section{Conclusión}

Se concluyó que los estudiantes de la carrera profesional de Educación de una universidad pública de Madre de Dios (Perú) se caracterizaban por presentar niveles moderados de burnout académico. Asimismo, se halló que algunas variables como el sexo, el grupo etario, la situación laboral y el año de estudio se asociaron significativamente con el burnout académico. En función a lo expuesto, es menester que el área de psicopedagogía de la universidad diseñe y ejecute programas que promuevan en los estudiantes el desarrollo de factores protectores para que puedan afrontar adecuadamente situaciones adversas y estresantes y de esta manera se disminuya la prevalencia del burnout académico.

Por otro lado, se recomienda a las futuras investigaciones que incrementen el número de la muestra, incluyendo a estudiantes de otras carreras profesionales, para poder generalizar los resultados y sería muy relevante que se asocien otras variables sociodemográficas y académicas con los factores del burnout académico (agotamiento emocional, cinismo y eficacia académica) para tener una mirada más precisa y comprender mejor esta problemática que no es ajena a los estudiantes universitarios.

\section{Referencias}

Aguayo, R., Cañadas, G., Assbaa, L., Cañadas, G., Ramírez, L. y Ortega, E. (2019). A risk profile of socio-demographic factors in the onset of academic burnout syndrome in a sample of university students. International Journal of Environmental Research and Public Health, 16(5), 707. https://doi.org/10.3390/ijerph16050707

Arias, W., Muñoz, A., Delgado, Y., Ortiz, M. y Quispe, M. (2017). Síndrome de burnout en personal de salud de la ciudad de Arequipa (Perú). Medicina y Seguridad del Trabajo, 63(249), 331-344. Recuperado de http://scielo.isciii.es/scielo.php?script=sci_arttext\&pid=S0465-546X2017000400331 
Bolaños, N. y Rodríguez, N. (2016). Prevalencia del Síndrome de Burnout académico en el estudiantado de Enfermería de la Universidad de Costa Rica. Revista Enfermería Actual en Costa Rica, 31, 1-19. http://dx.doi.org/10.15517/revenf.v0i31.24519

Caballero, C. (2006). Burnout, engagement y rendimiento académico entre estudiantes universitarios que trabajan y aquellos que no trabajan. Revista Psicogente, 9(16), 11-27. Recuperado de http://revistas.unisimon.edu.co/index.php/psicogente/article/view/2678

Caballero, C., Breso, E. y González, O. (2015). Burnout en Estudiantes Universitarios. Psicología desde el Caribe, 32(3), 424-441. Recuperado de http://rcientificas.uninorte.edu.co/index.php/psicologia/article/view/6217

Caballero, C., Gonzáles, O., Charris, N., Amaya, L. y Merlano, A. (2015). Relación del burnout académico con las estrategias de afrontamiento de estudiantes de enfermería en universidades privadas de la ciudad de Barranquilla. Tejidos Sociales, 1, 19-37. Recuperado de http://publicaciones.unisimonbolivar.edu.co/rdigital/ojs/index.php/tejsociales/article/view/1 $\underline{440}$

Caballero, C., Hederich, C. y Palacio, J. (2010). El burnout académico: delimitación del síndrome y factores asociados con su aparición. Revista Latinoamericana de Psicología, 42(1), 131146. Recuperado de http://www.scielo.org.co/scielo.php?pid=S0120$\underline{05342010000100012 \& \text { script }=\text { sci_abstract\&tlng=es }}$

Carlotto, M., Gonçalves, S. y Brazil, A. (2005). Predictores del Síndrome de Burnout en estudiantes de un curso técnico de enfermería. Diversitas, 1(2), 195-205. Recuperado de http://pepsic.bvsalud.org/scielo.php?script=sci_arttext\&pid=S1794-99982005000200008

Carrasco, S. (2006). Metodología de la Investigación Científica. Lima: Editorial San Marcos.

Correa, L., Loayza, J., Vargas, M., Huamán, M., Roldán, L. y Pérez, M. (2019). Adaptación Y Validación De Un Cuestionario Para Medir Burnout Académico En Estudiantes De Medicina De La Universidad Ricardo Palma. Revista de la Facultad de Medicina Humana, 19(1), 6473. http://dx.doi.org/10.25176/RFMH.v19.n1.1794

De la Rosa, G., Chang, S., Delgado, L., Oliveros, L., Murillo, D., Ortiz, R., Vela, G. y Yhuri, N. (2015). Niveles de estrés y formas de afrontamiento en estudiantes de Medicina en comparación con estudiantes de otras escuelas. Gaceta Médica de México, 151(4), 443-449. Recuperado de https://www.medigraphic.com/pdfs/gaceta/gm-2015/gm154c.pdf

Domínguez, S. (2018). Agotamiento emocional académico en estudiantes universitarios: ¿cuánto influyen las estrategias cognitivas de regulación emocional? Educación Médica, 19(2), 96103. https://doi.org/10.1016/j.edumed.2016.11.010

Estrada, H., De la Cruz, S., Bahamón, M., Pérez, J. y Cáceres, A. (2017). Burnout académico y su relación con el bienestar psicológico en estudiantes universitario. Espacios, 39(15), 7-23. Recuperado de https://www.revistaespacios.com/a18v39n15/18391507.html 
Freudenberger, H. (1974). Staff burnout. Journal of Social Issues, 30, 159-166. https://doi.org/10.1111/j.1540-4560.1974.tb00706.x

García, V., Vega, Y., Farias, B., Améstica, L. y Aburto, R. (2018). Factores asociados al burnout académico en estudiantes de internado profesional de Fonoaudiología. Ciencia \& Trabajo, 20(62), 84-89. https://dx.doi.org/10.4067/S0718-24492018000200084

Loayza, J., Correa, L., Cabello, C., Huamán, M., Cedillo, L., Vela, J., Pérez, L., Gonzáles, M. y De la Cruz, J. (2016). Síndrome de burnout en estudiantes universitarios: tendencias actuales. Revista de la Facultad de Medicina Humana, 16(1), 31-36. https://doi.org/10.25176/RFMH.v16.n1.333

Marenco, A., Suárez, Y. y Palacio, J. (2017). Burnout académico y síntomas relacionados con problemas de salud mental en universitarios colombianos. Psychologia, 11(2), 45-55. https://doi.org/10.21500/19002386.2926

Martínez, A. (2010). El síndrome de Burnout. Evolución conceptual y estado actual de la cuestión. Vivat Academia, 112, 42-80. https://doi.org/10.15178/va.2010.112.42-80

Maslach, C. (1982). Burnout: The cost of caring. New Jersey: Prentice-Hall Press

Maslach, C. y Jackson, S. (1981). MBI: Maslach Burnout Inventory. Manual. Palo Alto: University of California, Consulting Psychologists Press.

Merino, C. y Fernández, J. (2020). Ítem único de burnout académico: correlato con MBI-S en el nivel de los ítems. Educación Médica, 21(1), 61-62. https://doi.org/10.1016/j.edumed.2018.10.004

Núñez, L. (2017). Predictores del burnout académico en estudiantes de enfermería de las universidades nacionales de Áncash. Aporte Santiaguino, 10(1), 13-24. https://doi.org/10.32911/as.2017.v10.n1.179

Osorio, M., Parrello, S. y Prado, C. (2020). Burnout académico en una muestra de estudiantes universitarios mexicanos. Enseñanza e Investigación en Psicología, 2(1), 27-37. Recuperado de https://www.revistacneip.org/index.php/cneip/article/view/86

Rodríguez, J., Benavides, E., Ornelas, M. y Jurado, P. (2019). El burnout académico percibido en universitarios; comparaciones por género. Formación Universitaria, (12)5, 23-30. https://dx.doi.org/10.4067/S0718-50062019000500023

Rosales, Y. y Rosales, F. (2013). Burnout estudiantil universitario: Conceptualización y estudio. Salud mental, 36(4), 337-345. Recuperado de http://www.scielo.org.mx/scielo.php?script=sci_arttext\&pid=S0185-33252013000400009

Ruíz, C. (2002). Instrumentos y técnicas de investigación educativa. Houston: DANAGA. 
Sánchez, H., Reyes, C. y Mejía, K. (2018). Manual de términos en investigación científica, tecnológica y humanística. Lima: Universidad Ricardo Palma. Recuperado de http://repositorio.urp.edu.pe/handle/URP/1480

Schaufeli, W., Salanova, M., González, V. y Bakker, A. (2002). The Measurement of Engagement and Burnout: A Two Sample Confirmatory Factor Analytic Approach. Journal of Happiness Studies, 3, 71-92. https://doi.org/10.1023/A:1015630930326

Uribe, M. y Illesca, M. (2017). Burnout en estudiantes de enfermería de una universidad privada. Investigación en Educación Médica, 6(24), 234-241. https://doi.org/10.1016/j.riem.2016.11.005 